\title{
Modelling and Control of an Ultrasonic Motor: Application to a Mechanical Claw Drive
}

\author{
Frédéric Giraud, Betty Semail Member IEEE, Alain Bouscayrol Member IEEE \\ Laboratoire d'Électrotechnique et d'Électronique de Puissance de Lille, Polytech-Lille \\ Avenue Paul Langevin, 59655 Villeneuve d'Ascq Cedex \\ firstname.namedpolytech-Lille.fr \\ http://www.univ-lillel.fr/l2ep/
}

\begin{abstract}
Properties of the travelling Wave Ultrasonic Motor (TWUM) - among which we find a high-torque to mass ratio, locking without supply, ultrasonic operation - are very attractive for embedded application, such as human prothesis for example. But unfortunately, its control for high precision or high dynamic motion is not easy to achieve because of many non linearities induced by its particular energy conversion process. This is why this paper deals with the modelling of a TWUM, based on a causal approach. According to the inversion principle, control strategies are deduced as well as a novel torque estimation structure. A mechanical claw control using this torque estimator is then achieved and experimental results check the accuracy of the proposed control scheme.
\end{abstract}

\section{INTRODUCTION}

Travelling wave Ultrasonic Motors (TWUM) exploits a piezoelectrically generated flexural wave which propagates at the surface of a stator. This wave is able to propel by contact a rotor strongly pressed on the stator. The friction caused by the contact mechanism provides us with numerous advantages, including: breaking without supply, a high torque to mass ratio and high torque/low speed characteristics. Thus, while a speed reducer is often needed with an electromagnetic motor, it becomes useless in applications using TWUM, therefore leading to lightweight and compact applications. The last property is particularly important for embedded applications such as medical prothesis ones. Moreover, the working principle doesn't rely on magnetic field interactions which may be awkward for human uses.

Unfortunately, because of the mechanical non linearities, TWUMmodelling is not easy to achieve, especially in order to control its behavior, such as torque, speed or position.

This is due to the different energy conversions and more specifically to the complications caused by the contact mechanism between the stator and the rotor.

Several references have proposed modelling and control of the TWUM [1][2][3] The one used in this paper describes the real internal variables behavior. It will be first reminded and explained according to a causal formalism. Then, the control laws will be described following the inversion principles. For the particular application of an active claw control, a torque estimator will be explained. Experimental results will check the validity of the control laws as well as the modelling and estimator accuracy.

\section{Modelling And CONTROL OF THE TWUM}

\section{A. Modeling overview}

This section deals with a modelling we proposed for taking into account transitory operations [4][5]. This modelling relies on the assumption of a punctual contact between stator and rotor. Punctual means that the stator is in contact with the ideal rotor at one point only along a wavelength. Moreover, no sliding is considered between the ideal rotor and the stator, so both have the same instantaneous speed. On the figure 1, we have represented the travelling wave which propels the ideal rotor by only one point along a wavelength. In the fame fixed to the contact point $\left(\vec{u}_{r}, \vec{u}_{\theta}, \vec{u}_{z}\right)$ the travelling wave propagates toward the right (along $\vec{u}_{\theta}$ ) although the ideal rotor moves toward the left $\left(-\vec{u}_{\theta}\right)$. We also define the $\mathrm{z}$ axis about which the motor and the contact point turn. The location of the contact point is defined by the rotation angle $\theta_{c}$ measured from a fixed frame $(\mathrm{O}, \mathrm{x}, \mathrm{y})$.

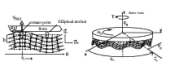

Fig. 1. View of the stator; under punctual contact assumption, the rotor is propelled by one point only along a wavelength.

1) Causal modelling in $(\alpha, \beta)$ frame: The TWUMis composed of a ring shaped stator on which small piezo-electric elements are bonded. These elements have alternate polling directions, and are divided into two groups, named phase $\alpha$ and phase $\beta$. These two phases are supplied by two independent voltages, $v_{\alpha}$ and $v_{\beta}$. Because of the piezo-electric effect, the first energy conversion stage converts these voltages into forces inside the stator; these forces bend the stator. And if the excitation frequency of the two supply voltages is sufficiently close to the resonant frequency of the stator, two stationary waves appear at its surface: the bending waves, whose height are named $w_{\alpha}$ and $w_{\beta}$. This second stage of the energy 
conversion can be described by the equation (1)

$$
\begin{aligned}
& m \ddot{w}_{\alpha}+d_{s} \dot{w}_{\alpha}+c w_{\alpha}=N v_{\alpha}-F_{r \alpha} \\
& m \ddot{w}_{\beta}+d_{s} \dot{w}_{\beta}+c w_{\beta}=N v_{\beta}-F_{r \beta}
\end{aligned}
$$

with

- $m$ the vibrating mass of the stator,

- $c$ the vibrating stiffness of the stator,

- $d_{s}$ the damping coefficient,

- $N$ the force factor.

In this equation, $F_{r \alpha}$ and $F_{r \beta}$ have been added to take into account the effect of external forces on the wave propagation. Their expression which depends on $F_{N}$ (the force pressing the rotor against the stator along the axis of the motor) and $F_{T}^{\prime}$ (the tangential force deriving from the load torque $T$ ) is quite difficult to determine, and strongly depends on the contact condition at the stator-rotor interface [2][6]. However, in the ideal case of a punctual contact condition, these forces are simply expressed using the location of the contact point $\theta_{c}$, and the well known rotational matrix, usually used in the study of electromagnetic machines. This calculation has been developed in [5], and derives from power balance of the system:

$$
\left(\begin{array}{c}
F_{r \alpha} \\
F_{r \beta}
\end{array}\right)=R\left(k \theta_{c}\right)\left(\begin{array}{c}
F_{N} \\
F_{T}^{\prime}
\end{array}\right) \quad R(\theta)=\left(\begin{array}{cc}
\cos (\theta) & -\sin (\theta) \\
\sin (\theta) & \cos (\theta)
\end{array}\right)
$$

with

$$
F_{T}^{\prime}=k \frac{h}{b^{2}} T
$$

And

- $h$ the thickness of the stator

- $b$ the radius of the rotor

- $k=\frac{2 \pi b}{\lambda}$ with $\lambda$ the wavelength of the excited mode.

The contact points are located at the travelling wave's crest. It can be shown that they are situated at an angle given by $w_{\alpha}$ and $w_{\beta}$ using the expression shown in (4)

$$
k \theta_{c}=\operatorname{atan}\left(\frac{w_{\beta}}{w_{\alpha}}\right)
$$

Moreover, as it can be seen in figure (1), the contact point speed which propels the rotor, has two components:

- $V_{N i d}$ along the axis of the motor, lifts up the rotor to enable the propagation of the travelling wave. During steady state, this speed is null.

- $V_{T i d}^{\prime}$ in the tangential direction, from which the rotational speed of the rotor $\omega_{i d}$ derives.

A kinetic study of the vibrating stator shows then that the expression of those speeds can be written using $\dot{w}_{\alpha \beta}$ and $R\left(k \theta_{c}\right)$ once again:

$$
\left(\begin{array}{c}
V_{N i d} \\
V_{\text {Tid }}^{\prime}
\end{array}\right)=R\left(-k \theta_{c}\right)\left(\begin{array}{c}
\dot{w}_{\beta} \\
\dot{w}_{\alpha}
\end{array}\right)
$$

The rotational speed of the ideal rotor $\omega_{i d}$ and $V_{T i d}^{\prime}$ is given by equation (6)[7]:

$$
\omega_{i d}=k \frac{h}{b^{2}} V_{T i d}^{\prime}
$$

However, the speed $\omega_{i d}$ does not actually correspond to the rotor speed, because the contact conditions observed at the stator-rotor interface are very different from those of the ideal contact conditions: in fact, because of friction, the actual rotational speed $\omega$ of the rotor decreases with the load torque $T$. Several models ([6][8][9]) exist to describe this behavior, but they are too complex to be taken into account in a control scheme. In order to achieve a straightforward modelling which may be useful for actuator control, a linear modelling for the global behavior of the motor can be proposed based on experimental results. This is the third energy conversion, given by equations (7).

$$
T=f_{0}\left(\omega_{i d}-\omega\right) \quad F_{N}=K_{N} \int\left(V_{N i d}-V_{N}\right) d t
$$

Of course, this process is very non linear, and the value of the parameters $f_{0}$ and $K_{N}$ should vary as a function of the operating point. However, we find here the key variables governing the evolution of the motor torque: the amplitude of the travelling wave, because $\omega_{i d}$ derives using it, and the rotational speed of the rotor itself. This roughly outlines the torque estimator described in [10] from the speed and the stator's deformation measurements.

In addition, the dynamic of the rotor, along the two degrees of freedom, leads to lay down two other equations:

$$
m_{r} \frac{d V_{N i d}}{d t}=F_{N}-F_{\tau} \quad J_{r} \frac{d \omega}{d t}=T-T_{r}
$$

Where:

- $m_{r}$ and $J_{r}$ are respectively the mass and rotational inertia of the rotor,

- $T_{r}$ is the external load torque applied on the rotor,

- $F_{\tau}$ is the normal force pressing the rotor against the stator.

Finally, all these equations yield a modeling which can be represented by way of an Energetic Macroscopic Representation $(E M R)$ as shown in figure 2. The $E M R$ is useful to highlight the energy conversion process and the electromechanical conversion. Following this formalism, the mechanical transformation processor are depicted with triangles, otherwise, the energy accumulators are described using barred rectangles.

2) Causal modelling in a rotating reference frame: the two rotational matrices, on the stationary waves (eq. 5) and on the forces (eq. 2), lead to setting down two new variables $V_{d}$ and $V_{q}$. As it is for the electromagnetic machines where current or voltages are expressed in a rotating reference frame fixed to the rotating electromagnetic field, $V_{d}$ and $V_{q}$ represent the supply voltages in a rotating reference frame fixed to the rotating travelling wave. So, this leads us to set down:

$$
\left(\begin{array}{c}
v_{\alpha} \\
v_{\beta}
\end{array}\right)=R\left(k \theta_{c}\right)\left(\begin{array}{c}
V_{d} \\
V_{q}
\end{array}\right)
$$

Using $V_{d}$ and $V_{q}$, equations $1,2,5$, are revised to produce two new equations respectively on $d$ axis and $q$ axis respectively, where the couplings are removed:

$$
m \dot{V}_{N i d}^{\prime}+d s V_{N i d}^{\prime}+\left(c-m k\left(\dot{\theta}_{c}\right)^{2}\right) \int V_{N i d}^{\prime} d t=N V_{d}-F_{N}^{\prime}
$$




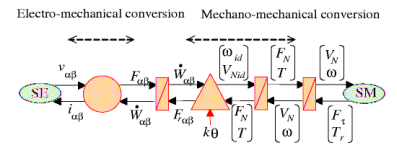

Fig. 2. EMR of the TWUM.

$$
2 m \dot{V}_{\text {Tid }}^{\prime}+d s V_{\text {Tid }}^{\prime}=N V_{q}-F_{T}^{\prime}
$$

In fact, in equation (11), the variables of the tangential axis appear only on the $q$ axis, though equation (10) shows that the normal variables only appear on the $d$ axis. This equation is of second order type, and completely describes the resonant behavior of the motor.

Upper part of figure 3 shows the EMR of the actuator in the rotating reference frame, reduced to the $q$ axis as it deals with the torque.

\section{B. Control strategies}

1) Maximal control structure: From EMR, it is easy to deduce the maximal structure of control, according to inversion principle. Transformation relations are directly inverted, but energetic storage elements are inverted using regulators. The lower part of figure 3 shows the speed control scheme as it may be deduced following these rules.

2) Non linearities considerations: The torque-speed relation of 7 has been approximated from the curves depicted at figure 4. The direct inversion of the relation is quite easy to achieve, but obviously may lead to an inaccurate controlled torque. To cope with this problem, two approaches are proposed: a torque regulation in a closed loop, or a global

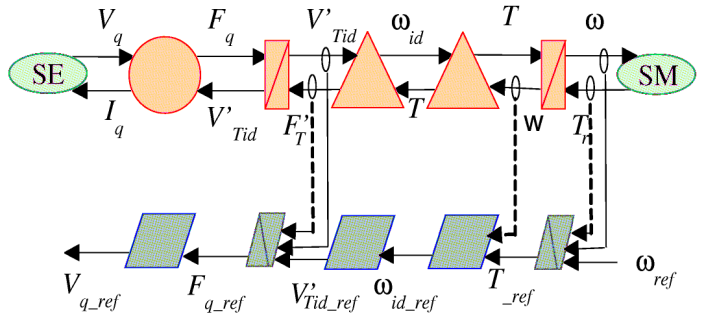

Fig. 3. EMR of the TWUM in the rotating reference frame - axis q only and the derived control scheme.

control including a behavior modelling regulator as detailed in [11]. Nevertheless, this last solution is available in case of

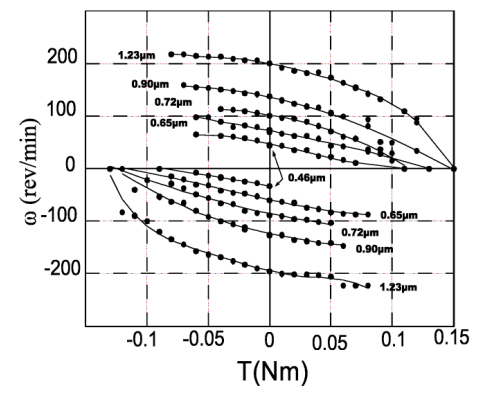

Fig. 4. Torque-speed characteristics of the TWUMfor given wave amplitude.

speed or position control. For torque control, a closed torque 
loop is necessary, as well as the knowledge of the actual output torque. In the specific application of a claw dedicated to a robot arm edge or a hand prothesis, the torque variations have to be monitored so as to indicate an object tightening for instance. The torque estimation may be achieved according to the modelling as explained in II-A.

3) The torque estimator: In order to avoid a speed sensor, the torque estimator will rely on equation 11. However, inverting this equation is not straightforward, because of the derivative term $2 m \dot{V}_{\text {Tid }}^{\prime}$, which should be approximated. A closed loop estimator could then be proposed, whose output would converge toward the solution. But considering a high performing estimator is required especially in the case of steady state operations - or slow variations of $V_{\text {Tid }}^{\prime}$, the problematic term $2 m \dot{V}_{\text {Tid }}^{\prime}$ is deleted in equation (11):

$$
d s V_{T i d}^{\prime}=N V_{q}-F_{T}^{\prime}
$$

Finally, using equations 3 in 12 leads to

$$
T=\frac{b^{2}}{k h}\left[N V_{q}-d_{s} V_{T i d}^{\prime}\right]
$$

Achieving the torque estimator.

\section{EXPERIMENTAL IMPLANTATION OF THE TORQUE ESTIMATOR[12]}

The TWUM used during the test is a Shinsei USR30 [13]. This motor has a $30 \mathrm{~mm}$ diameter and can provide a rated torque of $0.1 \mathrm{Nm}$ for a rated speed of $200 \mathrm{rev} / \mathrm{min}$. On the stator of this motor, an extra electrode is glued to the ring of piezo material. Due to the direct piezo-electrical conversion, the voltage measured on this sensor, named $v_{E A}$, is directly proportional to the deformation of the stator at this point. Unfortunately, this deformation is a combination of $w_{\alpha}$ and $w_{\beta}$ : thus it is difficult to build $\theta_{c}$ from the equation (4) because $w_{\alpha}$ and $w_{\beta}$ are not measured independently. In that case, measuring $V_{q}$ from $V_{\alpha}, V_{\beta}$ and (9) is impossible.

This leads us to set down the assumption that the amplitude of the two stationary waves are sinusoidal functions of time in quadrature, so:

$$
w_{\alpha}(t)=W \cos (2 \pi f t) \quad w_{\beta}(t)=W \sin (2 \pi f t)
$$

With $f$ the frequency of the supply voltages and $W$ the travelling wave's amplitude.

To fulfill this assumption, the construction of the actuator must be sufficiently careful to ensure a good positioning of the stator's electrodes on the piezo ring, thus avoiding dissymmetry between the two phases. However, this condition is often achieved in the Shinsei USR30 when it is supplied by two sinusoidal voltages in quadrature with the same amplitude:

$$
v_{\alpha}(t)=V \cos (2 \pi f t+\Psi) \quad v_{\beta}(t)=V \sin (2 \pi f t+\Psi)
$$

with $V$ the amplitude of supply voltages, and $\Psi$, the phase difference between $v_{\alpha}(t)$ and $w_{\alpha}(t)$. On the experimental test bench, $V$ is kept constant, though $f$ varies.

Then, equations (4) and (14) yield to:

$$
\theta_{c}=\operatorname{atan} \frac{W \sin (2 \pi f t)}{W \cos (2 \pi f t)}=2 \pi f t
$$

To complete the identification of $T$ from equation (13), it is necessary to know $V_{\text {Tid }}^{\prime}$, but however this is an internal variable. It may be calculated thanks to equation (5), and from the measurement of $\dot{w}_{\alpha}$ and $\dot{w}_{\beta}$. Once again, to reach these values, we use the previous assumption which leads to equation (14). Then, we find the relationship between the wave amplitude and the tangential speed of the rotor, using equations (16) and the rotational matrix (5):

$$
\begin{aligned}
V_{\text {Tid }}^{\prime} & =-\dot{w}_{\alpha} \sin (2 \pi f t)+\dot{w}_{\beta} \cos (2 \pi f t) \\
& =(2 \pi f) W
\end{aligned}
$$

Therefore, $V_{\text {Tid }}^{\prime}$ is proportional to the travelling wave's amplitude. Now, W can be deduced by measuring the voltage amplitude of $v_{E A}$; the different state variables of the torque estimator can then be measured. But parameters appearing in the equation (13) have also to be identified.

\section{A. Measurement of the stator parameters}

1) Identification of $d_{s}$ : For an unloaded motor which has reached its steady state, the travelling wave's amplitude $W$ derives from the equation 13 where $T$ is set to 0 . In these conditions:

$$
d_{s} 2 \pi f W=N V_{q}
$$

Identifying $d_{s}$ can then be achieved by keeping $V$ constant; plotting the evolution of $W$ against $V q$ should result in a straight line, whose slope gives the ratio $\frac{N}{d_{s} 2 \pi f}$.

However, this method is quite sensitive to non linearities which induce variations of $d_{s}$ with $W$ [14]. This is why, instead of $V, W$ is kept constant. So, for each value of $V_{q}, V$ is tuned in order to have $W$ equal to a value we fixed before.

It appears that $d_{s}$ depends more on the operating wave amplitude $W$ and less on the rotational direction. In order to take into account this non linearity in the torque estimator, we are led to representing in figure 5 the evolution of $d_{s}$ in $W$, or rather in $\frac{1}{W}$ because this results in a linear shape.

Equation 19 then fits this curve:

$$
d_{s}=-d_{s 0}+\frac{\rho}{W}
$$

The values of $d_{s 0}$ and $\rho$ are obtained from figure 5:

$$
d_{s 0}=19.2 N . s . m^{-1} \quad \rho=47.8 .10^{-6} N . s
$$

So, taking into account the non-linearity of $d_{s}$, the torque estimator can be achieved by merging the equations 13, 17 and 19:

$$
T=\frac{b^{2}}{k h}\left(d_{s 0} 2 \pi f W+N V q-2 \pi f \rho\right)
$$

2) Identification of $k \frac{h}{b^{2}}$ : The identification of this parameter derives once again from equation (13). But now, $T$ will vary and $V_{q}$ is adjusted to keep $W$ constant. So, the evolution of $V_{q}$ in $T$ should be a straight line whose slope is $k \frac{h}{N b^{2}}$. We represents in figure ?? the result curves for several values of $W$.

The results show that the experimental trials and the modelling are consistent, since the curves are nearly straight. However, the slope of these lines depends on the level of the 


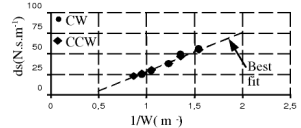

Fig. 5. Evolution of $d_{s}$ as a function of $\frac{1}{W}$ for both rotational direction and fitting.

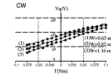

Fig. 6. $V_{q}$ as a function of $T$ and comparison with a straight line.

stator's deformation $W$ and also on the direction of rotation. These variations reveal non-linearities in the modelling; in fact, they express the limits of this modelling, and more specifically the limit imposed by the assumption that there is only one contact point. This is why, an average value of $k \frac{h}{b^{2}}$ is then calculated from the trials, and the slope of the curves drawn on figure 6 yielding $k \frac{h}{b^{2}}=67.2 m^{-1}$.

\section{B. Experimental runs}

Different trials have been carried out to illustrate the performance of this torque estimator. The first measurements shown in figure 7(a) depict the evolution of the estimated load torque and the measured load torque. During this trial, the stator wave's amplitude was kept constant $(W \simeq 0.5 \mu m$ ) while the load torque $T$ was time varying.

The second measurement of figure 7(b) shows the evolution of the measured load torque compared to its estimation when $W$ was time varying.

Considering that the torque estimator is quite straightforward, the estimated load torque is found to be consistent with the measurements of the experimental trials of figures 7(b) and 7(a). Estimation errors can however be measured on figure 7(b), revealing that the estimation depends on $W$. More obviously, errors are amplified for braking operations while they are acceptable for other conditions. This is mainly due to the averaged value of $k \frac{h}{b^{2}}$ we had to use in equation 20 and which is a tradeoff between all the values identified for all the operating points of the chapter III-A. Performances of the estimator could then be improved by taking into account the variation of $k \frac{h}{b^{2}}$ in $W$, but this would be achieved at the expense of complexity. Despite this problem, applications can take advantage of this torque estimator, and this is dealt with in the next section.

\section{APPLICATION: FORCE CONTROL OF TWUM ACTUATED CLAW}

\section{A. Working principle of a claw}

Because of torque vs speed characteristics, Travelling Wave Ultrasonic Motors are very suitable for applications which need a low speed but a high torque. In this field of applications, the electrically actuated claws are devices which are widely used, for example at the end of a robot arm to allow object prehension.

Different controls of the claw can be achieved. The first control type is a position control of the actuator of the claw between an opened position till the closed position. This is well suited for objects which all have the same dimensions. But if variable objects of random sizes have to be picked up, the larger objects are held extremely tight, while the smallest ones are not even caught (figure 8).

This is why, instead of having a claw position control, a force limitation is preferred. In this operating mode, the claw closes up at a constant speed until the force at the claw 's tip reaches a limited value.

This last control method is quite easy to achieve with electromagnetic motors, because the torque is proportional to the supply current which is often controlled, and can thus be limited. But those motors have high speed characteristics, so they need a speed reducer in order to adapt the output speed of the shaft to the desired speed of the claw when closing. This increases the system's bulk and its cost.

In this application, a TWUM is interesting, because it can be directly mounted on the claw since its low-speed characteristic is well adapted to the system. The torque estimator developed in this article is implemented to ensure the limitation of the tightening force.

The control scheme is depicted in figure 9. The torque estimator is depicted and its output is used as an input for a strategy block. This strategy block is inserted to determine the reference wave amplitude: below the limit torque value, 


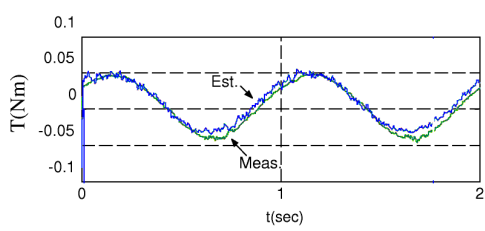

(a) For a constant wave's amplitude.
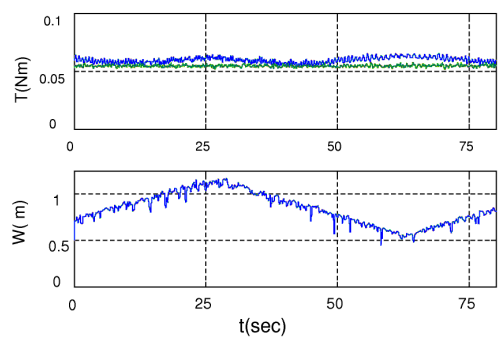

(b) For a variable wave's amplitude

Fig. 7. Comparison between the actual load torque and the estimator's output. Experimental runs.
Fig. 8. Effect of a controlled position claw when the object is sufficiently big, and in the case where it is too small.

the wave amplitude reference is kept constant; when the torque limit is reached, the wave amplitude is set to zero, so as to lock the claw.

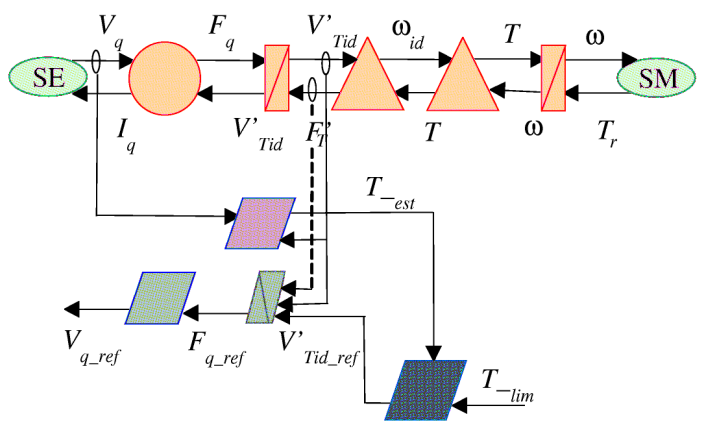

Fig. 9. Control structure for an active claw application.

The next section presents the experimental runs of the claw actuated by a TWUM, with its torque limitation.

\section{B. Experimental runs}

The claw fixed on the TWUM's shaft is depicted in figure 10. Several objects with different sizes can be positioned, and for each run, the internal variable of the motor can be recorded. Thus, figure 11 depicts the evolution of $V_{q}$, the motor rotational angle, the measured and estimated torque and finally the wave amplitude $W$ - which is also controlled. These curves are obtained with $T_{l i m}=0.05 \mathrm{Nm}$. The cycle begins at $t=0$ with the starting signal; a wave amplitude reference of about 


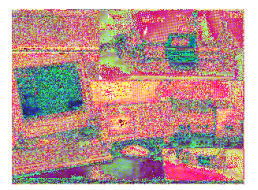

Fig. 10. The experimental Setup.

$1 \mu \mathrm{m}$ is ordered, and the claw closes. The estimated torque as well as the measured one are initially null, but at $t=50 \mathrm{~ms}$ they both start to increase because the claw is in contact with the object to be grasped. Consequently, $V_{q}$ increases in accordance equation (20), since $W$ is kept constant.

At $t=85 \mathrm{~ms}$, the estimated torque reaches the limitation: a reference value of $W$ of $0 \mu \mathrm{m}$ is ordered which is obtained in almost $10 \mathrm{~ms}$. During this short time, the torque actually applied to the claw can be a little larger due to the time the wave amplitude needs to become null. Then the torque estimator is switched off because the very low wave amplitude leads to erroneous estimated torque values.

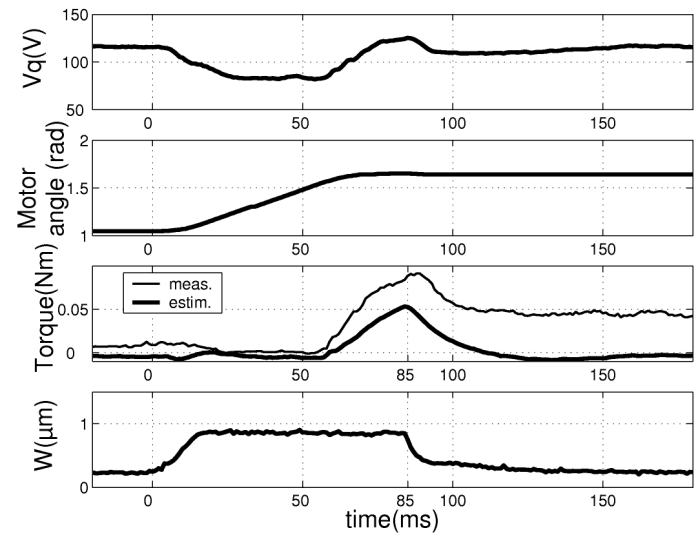

Fig. 11. Internal variables of the motor for a closing sequence of a TWUM actuated claw, with limitation of the torque.

So, the claw stops its grasping action when it meets an object. This contact is detected through an increasing in the motor's torque, which is estimated on line. Of course, the force with which the object is taken depends on the value of $T_{l i m}$.

\section{CONCLUSION}

In this article, we have presented a method to achieve a TWUMcontrol for a mechanical claw function application. According to he causal modelling of the actuator, several control structures have been described. More specifically, we have developed a torque estimator in order to achieve the torque monitoring for a tightening detection. This estimator relies on the causal modelling of the motor which helps to deduce the estimator's equation. Compared to the torque estimators which rely on the torque/speed characteristics of the motor, we emphasize that the estimator presented here doesn't need the measurement of the rotational speed of the motor; this allows to remove the speed sensor, and the measurements have confirmed the global structure of the modelling, while the estimator is successfully tested on the claw application in the final section.

Future work should increase the precision of this torque estimator, more specifically at low rotational speeds, to allow sensorless torque regulators. This implies the control of voltages phases leading to the loss of quadrature. A new modelling should be introduced to take into account this voltage supply.

\section{ACKNOWLEDGMENT}

This work has been carried out within the framework of the INRIA Alcove project and is supported by the IRCICA (Institut de Recherche sur les Composants logiciels et matériels pour l'Information et la Communication Avancée).

\section{REFERENCES}

[1] J.Maas, T.Schulte, N.Fröhleke. Model based control for ultrasonic motors. IEEE trans. on Ultrasonics, Ferroelectrics and Frequency control, 5:165-180, june 2000.

[2] W. Hagood IV, A.J. McFarland. Modeling of a piezoelectric rotary ultrasonic motor. IEEE Transactions on ultrasonics, ferroelectrics and frequency control, 42(2):210-224, march 1995.

[3] N. El ghouty. Hybrid modeling of a traveling wave piezo electric motor. $\mathrm{PhD}$ thesis, Aalborg University, department of control engineering, May 2000.

[4] F.Giraud, B.Semail, J.-T.Audren. Analysis and phase control of a piezo electric travelling wave ultrasonic motor for haptic stick application. IEEE trans. on Industry applications, 40:1541-1549, nov-dec 1997.

[5] F.Giraud, B.Lemaire-Semail. Causal modeling and identification of a travelling wave ultrasonic motor. European Physical Journal of Applied Physics, 21:151-159, february 2003.

[6] J.Maas, P.Ide, N.Fröhleke, H.Grostollen. Simulation model for ultrasonic motors powered by resonant converters. IAS'95, 1:111-120, October 1995.

[7] Toshiiku Sashida, Takashi Kenjo. An introduction to Ultrasonic Motors. Clarendon Press, 1993.

[8] H.Hirata, S.Ueha. Characteristics estimation of a travelling wave type ultrasonic motor. IEEE trans. on Ultrasonics, Ferroelectrics and Frequency control, 40:402-406, july 1993.

[9] T.Maeno, T.Tsukimoto, A;Miyake. Finite element analysis of the rotor/stator contact on e ring-type ultrasonic motor. IEEE trans. on Ultrasonics, Ferroelectrics and Frequency control, 39:668-674, july 1993.

[10] J.Maas, T. Schulte, H.Grostollen. Optimized drive control for inverterfed ultrasonic motors. IEEE Industry applications society ( IAS'97), 1:690-698, october 1997. 
[11] F.Giraud, B.Lemaire-Semail, Julien Aragones, Jacques Robineau, JeanThierry Audren. Precise position control of a travelling wave ultrasonic motor. Procceding of the 2005 IEEE Industry Applications conference, 40th annual meeting - Kowloon - Hong Kong -, October 2005.

[12] F.Giraud, B.Lemaire-Semail. A torque estimator for a travelling wave ultrasonic motor - application to an active claw. IEEE trans. on Ultrasonics, Ferroelectrics and Frequency control, to be published 2006.

[13] ShinseI. Internet web site. http://www.tky.3web.ne.jp/rusrmotor/English /html/index.html.

[14] J.Maas, H.Grostollen. Averaged model of inverter-fed ultrasonic motors. IEEE power electronics specialists conf. ( PESC ), 1:740-786, june 1997. 